Article

\title{
Pulsed Laser Deposition of Bismuth Vanadate Thin Films-The Effect of Oxygen Pressure on the Morphology, Composition, and Photoelectrochemical Performance
}

\author{
Konrad Trzciński ${ }^{1, *}$, Mariusz Szkoda ${ }^{1}$, Maria Gazda ${ }^{2}$, Jakub Karczewski ${ }^{2}{ }^{\circledR}$, Adam Cenian ${ }^{3}$, \\ Galina M. Grigorian ${ }^{4}$ and Mirosław Sawczak ${ }^{3}$ (D) \\ 1 Faculty of Chemistry, Gdansk University of Technology, Narutowicza 11/12, 80-233 Gdansk, Poland; \\ marszko@pg.edu.pl \\ 2 Faculty of Applied Physics and Mathematics, Gdansk University of Technology, Narutowicza 11/12, 80-233 \\ Gdansk, Poland; maria.gazda@pg.edu.pl (M.G.); jakub.karczewski@pg.edu.pl (J.K.) \\ 3 Center for Plasma and Laser Engineering, The Szewalski Institute of Fluid-Flow Machinery, Fiszera 14, \\ 80-231 Gdansk, Poland; cenian@imp.gda.pl (A.C.); mireks@imp.gda.pl (M.S.) \\ 4 Physical Department, St. Petersburg State University, 199034 St. Petersburg, Russia; galgr2@rambler.ru \\ * Correspondence: kontrzci@pg.edu.pl
}

Received: 13 February 2020; Accepted: 13 March 2020; Published: 17 March 2020

\begin{abstract}
Thin layers of bismuth vanadate were deposited using the pulsed laser deposition technique on commercially available FTO (fluorine-doped tin oxide) substrates. Films were sputtered from a sintered, monoclinic $\mathrm{BiVO}_{4}$ pellet, acting as the target, under various oxygen pressures (from 0.1 to 2 mbar), while the laser beam was perpendicular to the target surface and parallel to the FTO substrate. The oxygen pressure strongly affects the morphology and the composition of films observed as a $\mathrm{Bi}: \mathrm{V}$ ratio gradient along the layer deposited on the substrate. Despite $\mathrm{BiVO}_{4}$, two other phases were detected using XRD (X-ray diffraction) and Raman spectroscopy- $\mathrm{V}_{2} \mathrm{O}_{5}$ and $\mathrm{Bi}_{4} \mathrm{~V}_{2} \mathrm{O}_{11}$. The V-rich region of the samples deposited under low and intermediate oxygen pressures was covered by $\mathrm{V}_{2} \mathrm{O}_{5}$ longitudinal structures protruding from $\mathrm{BiVO}_{4}$ film. Higher oxygen pressure leads to the formation of $\mathrm{Bi}_{4} \mathrm{~V}_{2} \mathrm{O}_{11} @ \mathrm{BiVO}_{4}$ bulk heterojunction. The presented results suggest that the ablation of the target leads to the plasma formation, where Bi and V containing ions can be spatially separated due to the interactions with oxygen molecules. In order to study the phenomenon more thoroughly, laser-induced breakdown spectroscopy measurements were performed. Then, obtained electrodes were used as photoanodes for photoelectrochemical water splitting. The highest photocurrent was achieved for films deposited under $1 \mathrm{mbar}_{2}$ pressure and reached $1 \mathrm{~mA} \mathrm{~cm}^{-2}$ at about $0.8 \mathrm{~V}$ vs $\mathrm{Ag} / \mathrm{AgCl}(3 \mathrm{M} \mathrm{KCl})$. It was shown that $\mathrm{V}_{2} \mathrm{O}_{5}$ on the top of $\mathrm{BiVO}_{4}$ decreases its photoactivity, while the presence of a bulk $\mathrm{Bi}_{4} \mathrm{~V}_{2} \mathrm{O}_{11} @ \mathrm{BiVO}_{4}$ heterojunction is beneficial in water photooxidation.
\end{abstract}

Keywords: bismuth vanadate; pulsed laser deposition; photoelectrochemistry

\section{Introduction}

Bismuth vanadate is one of the most promising candidates for visible light photoelectrochemical water splitting [1]. It is characterized by a moderated energy band gap [2] and high absorption coefficient [3] allowing material to be efficiently excited by visible light. The appropriate flat band potential makes $\mathrm{BiVO}_{4}$ a good candidate as a photoanode for efficient water oxidation [4]. It was estimated that $\mathrm{BiVO}_{4}$ illuminated by AM1.5 solar light should generate $7.5 \mathrm{~mA}$ per $\mathrm{cm}^{2}$ [5]. The theoretical efficiency of water photooxidation has not been achieved yet. This is related to the adverse 
phenomena such as bulk $\mathrm{e}^{-} / \mathrm{h}^{+}$recombination due to the poor hole mobility and recombination on the surface states. There are many literature reports about $\mathrm{BiVO}_{4}$ modifications that lead to the enhancement of its performance [6]. The most common ways consists of Mo and $\mathrm{W}$ doping [7] and heterostructures formation [8]. Up to now, the highest photocurrent densities were achieved for bulk (i) W-doped $\mathrm{BiVO}_{4} / \mathrm{V}_{2} \mathrm{O}_{5}$ heterojunction $\left(6.6 \mathrm{~mA} \mathrm{~cm}^{-2}\right)$ and (ii) electrode material that was prepared on a scaffold of polystyrene spheres scaffold and consisted of $\mathrm{WO}_{3}$ and molybdenum doped $\mathrm{BiVO}_{4}$ nanoparticles $\left(5.8 \mathrm{~mA} \mathrm{~cm}^{-2}\right)$, both covered by a $\mathrm{FeOOH} / \mathrm{NiOOH}$ dual layer catalyst $[9,10]$. However, the theoretical value of the photocurrent should be possible to achieve for "bare" $\mathrm{BiVO}_{4}$. Thus, it is still worthwhile to focus on the optimization of $\mathrm{BiVO}_{4}$ synthesis and layer preparation. In the literature, one can find different techniques to obtain bismuth vanadate films acting as photoelectrodes, e.g., electrostatic spray pyrolysis [11], deposition of inverse opals using polystyrene spheres as a template [12], simple dip-coating method [13], polymer-assisted spin coating [14], and most promising pulsed laser deposition technique (PLD) [15]. The PLD is a common method of photoactive material deposition [16]. The sputtering conditions during PLD strongly affect the properties of the resulting layer [17]. It was shown that pulsed laser deposited $\mathrm{BiVO}_{4}$ films on FTO substrates obtained at $230{ }^{\circ} \mathrm{C}$ can generate high photocurrents equal to about $3 \mathrm{~mA} \mathrm{~cm}{ }^{-2}$, but only in the hole-scavenger containing electrolyte [18]. Other authors showed that the high temperature $\left(500^{\circ} \mathrm{C}\right)$ in the deposition chamber makes the growth of $\mathrm{BiVO}_{4}$ crystallites anisotropic [19]. The specific orientation and (001) facet exposition facilitates the photoexcited charge carriers transport through the layer and enhance the efficiency of photocurrent generation. A similar effect of $\mathrm{BiVO}_{4}$ anisotropic growth was observed for the films deposited at $550^{\circ} \mathrm{C}$ on the yttria-stabilized zirconia (YSZ), however the activity of the electrodes in water photooxidation reaction was unsatisfactory [20]. Since the ambient gas pressure strongly affects the expansion dynamic of plasma plume, one may expect different properties of films deposited under different pressures [21]. Indeed, it was reported that the PLD technique can be applied in order to form type-II heterojunction $\left(\mathrm{BiVO}_{4} / \mathrm{Bi}_{4} \mathrm{~V}_{2} \mathrm{O}_{11}\right)$ on specifically oriented substrates using stoichiometric $\mathrm{BiVO}_{4}$ as a target and the composition of a resulting film can be tuned by changing oxygen pressure [22]. The presence of oxygen in the deposition chamber is crucial to obtain metal oxides films using PLD. The deviations from stoichiometry were observed, e.g., for $\mathrm{ZnO}$ [23]. Authors showed that $\mathrm{Zn}: \mathrm{O}$ ratio was close to one only in the case of the deposition performed in oxygen atmosphere, and the oxygen depletion was observed for process performed in Ar and in vacuum. Oxygen deficiency was also reported for $\mathrm{SiO}_{2}$ films deposited in vacuum [24]. Thus, in order to avoid this effect, all depositions of $\mathrm{BiVO}_{4}$ layers were carried in oxygen atmosphere. Some authors claimed that the excess of $\mathrm{Bi}$ in the target is necessary to get stoichiometric $\mathrm{BiVO}_{4}$ films on YSZ substrates [25]. The PLD technique carried out by sequential ablation was also utilized in order to prepare $\mathrm{BiVO} / \mathrm{WO}_{3} / \mathrm{SnO}_{2}$ heterojunction, however deposition parameters were not thoroughly investigated [26].

This work is focused on the influence of oxygen pressure in the chamber during pulsed laser deposition on the composition, morphology, and photoelectrochemical properties of bismuth vanadate. Bismuth vanadate layers were deposited on the commercially available FTO substrates from $\mathrm{BiVO}_{4}$ pellet (equimolar amounts of bismuth and vanadium). The substrate was placed in the chamber parallel to the laser beam. Such geometry is not chosen in order to obtain the highest possible photoelectrochemical activity of the deposited layer. The proposed geometry allows the spatial distribution of elements deposited on the substrate to be investigated. The results obtained from such geometry of the system give new insight into the mechanism of bismuth vanadate ablation from stoichiometric $\mathrm{BiVO}_{4}$ targets. The resulting films were characterized using XRD, Raman spectroscopy, energy dispersive $\mathrm{X}$-ray spectroscopy, and scanning electron microscopy. The photoelectrochemical measurements were performed in an aqueous electrolyte under AM1.5 simulated solar illumination in order to investigate the influence of deposition parameters on the efficiency of photoelectrochemical water oxidation. 


\section{Materials and Methods}

\subsection{Preparation and Characterization of the Target}

Bismuth vanadate targets were prepared via conventional solid state reaction using $\mathrm{NH}_{4} \mathrm{VO}_{4}$ (Sigma) and $\mathrm{Bi}\left(\mathrm{NO}_{3}\right)_{3} \cdot 6 \mathrm{H}_{2} \mathrm{O}$ (Sigma) as precursors. Equimolar amounts of $\mathrm{Bi}$ and $\mathrm{V}$ sources were ground in a mortar and pestle. The mixture was dried overnight at $100{ }^{\circ} \mathrm{C}$ and then pressed into a cylindrically shaped pellet with diameter $10 \mathrm{~mm}$ using hydraulic press (Specac) with a load of about 64 MPa for about $120 \mathrm{~s}$. The pellet was annealed in static air at $500{ }^{\circ} \mathrm{C}$ (tubular furnace, Czylok) in order to transform precursors into $\mathrm{BiVO}_{4}$ and get rid of $\mathrm{NH}_{4} \mathrm{NO}_{3}$ formed during the reaction. Then, the material was reground and mixed with poly(ethylene oxide), (Sigma, Mw 300,000) in a mass ratio 50:1 $\left(\mathrm{BiVO}_{4}: \mathrm{PEO}\right)$. The polymer (PEO) facilitates the formation of mechanically stable pellets obtained using a hydraulic press (32 MPa, $120 \mathrm{~s}$ ). These prepared pellets were sintered at $700{ }^{\circ} \mathrm{C}$ for $10 \mathrm{~h}$ in order to prepare a target for pulsed laser deposition. The high temperature causes decomposition of polymeric binder and the resulting target consists of pure $\mathrm{BiVO}_{4}$. The crystal structure of the targets was studied using XRD technique. As is shown in Figure 1a, the proposed method of target preparation leads to the formation of single-phase monoclinic $\mathrm{BiVO}_{4}$. Moreover, the Raman spectroscopy technique confirmed that $\mathrm{BiVO}_{4}$ pellet is free from carbon residues coming from a binder, see Figure $1 \mathrm{~b}$. All bands present in Raman spectrum originate from $\mathrm{BiVO}_{4}$ structure. Peaks at Raman shifts below 250 $\mathrm{cm}^{-1}$ can be prescribed to lattice modes. Bands at $320-370 \mathrm{~cm}^{-1}$ range and $680-915 \mathrm{~cm}^{-1}$ range are coming from $\mathrm{V}-\mathrm{O}$ bending and stretching vibrations, respectively. The morphology of the $\mathrm{BiVO}_{4}$ target was examined using SEM. The SEM micrograph of the annealed target is presented in Figure 1c. It can be seen that the pellet is homogenous and contains irregular particles of $\mathrm{BiVO}_{4}$ with diameters in a range from about 3 to $22 \mu \mathrm{m}$.
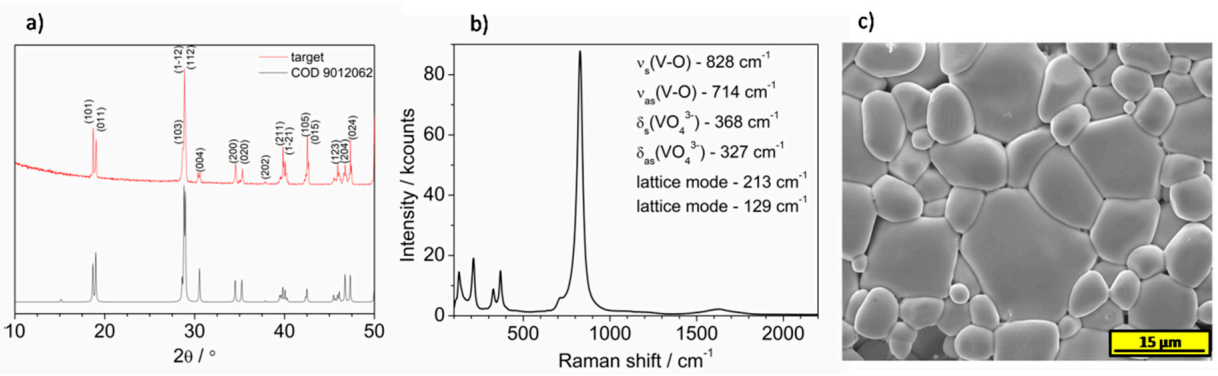

Figure 1. (a) The comparison of XRD patterns of target and reference pattern of $\mathrm{BiVO}_{4}$ (from crystallography open database 9012062). (b) The Raman spectrum of $\mathrm{BiVO}_{4}$ target. (c) SEM micrograph of annealed $\mathrm{BiVO}_{4}$ target.

\subsection{Layers Deposition}

The bismuth vanadate films were deposited in the specially developed PLD chamber under conditions of controlled temperature and process gas pressure. The scheme of the deposition chamber is shown in Figure 2a. The target (3) and substrate (4) are oriented perpendicularly to each other and mounted in a quartz vacuum chamber (2). The temperature inside the vacuum chamber is controlled with the cylindrical heating system (1) and the temperature control unit (8). The temperature during the deposition $\left(500^{\circ} \mathrm{C}\right)$ is controlled by means of a k-type sensor mounted inside the vacuum chamber. The ablation process is performed using a $266 \mathrm{~nm}, 6 \mathrm{~ns}$ pulse-duration laser-beam of $\mathrm{Nd}$ : YAG laser equipped with a FHG module (Brilliant B, Quantel, Newbury, UK). The energy density of the laser was established at about $2.5 \mathrm{~J} \mathrm{~cm}^{2}$. The laser beam is focused on the target surface with the quartz lens (6) mounted on the xy stage to allow scanning of the target surface and avoidance of crater formation. The gas pressure and composition are controlled by means of a vacuum pump and gas dosing valve (7). The pressure of the oxygen inside the deposition chamber was adjusted in the range from 0.1 to 2 mbar depending on the deposition-process conditions. The self-made system for pulsed laser deposition is 
presented in Figure S1. A part of the samples was prepared in order to measure the gradient of the films thickness (a part of the substrate parallel to the plasma plume was covered). The thickness of the films was estimated using the DektakXT profilometer (Bruker, Billerica, MA, USA). The thickness of the layers did not change significantly with oxygen pressure, see Table S1 in Supporting Information. The results from the profilometer were also confirmed by a cross-sectional SEM micrograph. The exemplary image is presented in Figure S2. It can be concluded, that the thickness of the film roughly equals to the size of single crystallite. Atomic force microscopy (AFM) measurements were performed using Easy Scan 2 microscope (Nanosurf, Liestal, Switzerland) working in the contact mode in order to investigate the topography of deposited films. Films were prepared under different oxygen pressures: $0.1,0.25,0.5,1$, and 2 mbar. All samples were deposited at $500^{\circ} \mathrm{C}$ for $15 \mathrm{~min}$. Degreased FTO substrates were placed $5 \mathrm{~mm}$ from the target parallel to the laser beam. The area of the deposited layers was equal to $0.56 \mathrm{~cm}^{2}(0.8 \mathrm{~cm} \times 0.7 \mathrm{~cm})$.

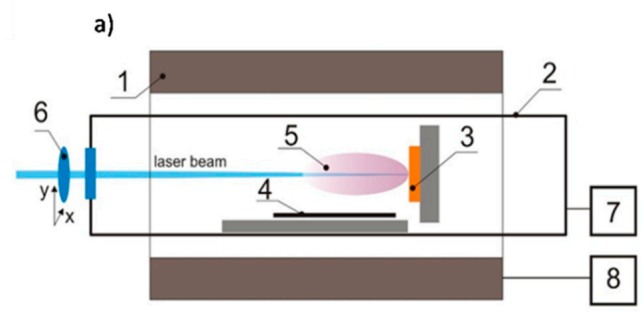

b)

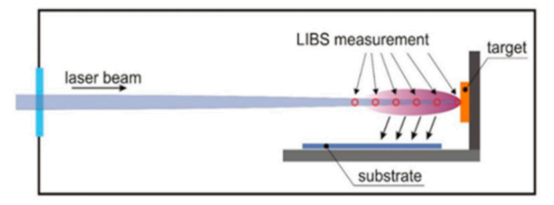

Figure 2. (a) Scheme of the pulsed laser deposition technique (PLD) setup: (1) heating chamber, (2) quartz process chamber, (3) target, (4) substrate, (5) laser ablation plume, (6) focusing lens mounted on xy stage, (7) pressure and process gas-composition control-unit, and (8) temperature controller. (b) Scheme of the pulsed laser deposition zone with marked positions for laser-induced breakdown spectroscopy (LIBS) measurements.

\subsection{Plasma Analysis}

In order to study the processes occurring in the laser-induced plasma, especially ionization and recombination processes of ablated species during interaction with oxygen molecules, the LIBS (laser-induced breakdown spectroscopy) measurements were undertaken. The detection of plasma emission was realized using $0.3 \mathrm{~m}$ monochromator (SR303i, Andor, Belfast, UK) equipped with 600 grooves/mm grating and intensified charge-coupled device (i-CCD) detector (DH740, Andor). Spectra were recorded in the range of 260-800 nm. Each spectrum was recorded in time resolved mode and averaged from 100 acquisitions. The plasma emission was recorded at different distances from target $(0,5,15,15$ and $20 \mathrm{~mm})$ as shown in Figure $2 \mathrm{~b}$.

\subsection{Apparatus}

Raman spectroscopy measurements were performed using a InVia spectrometer (Renishaw, Wotton-under-Edge, UK) with green laser excitation $(514 \mathrm{~nm})$ using a $100 \times$ objective and $5 \%$ of total laser power. The measurement spot was round-shape with diameter of about $2 \mu \mathrm{m}$. XRD patterns were recorded using the diffractometer (Xpert PRO-MPD, Philips, Amsterdam, The Netherlands) with copper $\mathrm{K} \alpha$ radiation $(8.04 \mathrm{keV})$. The morphology of the samples was investigated by Schottky field emission scanning electron microscopy (Quanta FEG 250, FEI, Hillsboro, OR, USA) with an ET (Everhart-Thornley) secondary electron detector. The beam accelerating voltage was kept at $10 \mathrm{kV}$ for films and $20 \mathrm{kV}$ for targets analysis. 


\section{Results and Discussion}

The prepared films were characterized using an XRD technique. As it is shown in Figure 3, all patterns exhibits typical reflection characteristic for monoclinic bismuth vanadate. Recently, it was reported that deposition temperature significantly affects the orientation of $\mathrm{BiVO}_{4}$ crystallites growing on the FTO substrates [19]. Authors showed that oxygen pressure does not change significantly the crystal structure of resulting layers. Indeed, the presented diffractograms differ rather slightly. Differences may result i.a. from the different thicknesses of the layers and the relative intensity of peaks coming from the FTO substrate. Patterns consist of all the reflexes characteristic for the monoclinic $\mathrm{BiVO}_{4}$ phase. However, in the case of the sample deposited under $0.5 \mathrm{mbar}$, reflex at about $15.5^{\circ}$ was detected $(\mathrm{V})$. It could be related to the (002) plane of $\mathrm{BiVO}_{4}$ suggesting anisotropic growth of the crystallites, but the intensity of the peak recorded at $30.5^{\circ}$ coming from (004) plane was not enhanced. Thus, it is very likely that it is coming from $\mathrm{V}_{2} \mathrm{O}_{5}(020)$ plane [27]. The exemplary XRD pattern recorded in a small-angle scattering mode is shown in Figure $\mathrm{S} 3$ for the samples deposited at 0.1, 0.5, and 2 mbar. The patterns are characterized by a much lower intensity of reflections from the FTO substrate as expected. Generally, the same reflexes have been recorded as in the case of conventional XRD analysis. However, the reflection coming from $\mathrm{V}_{2} \mathrm{O}_{5}(010)$ plane at $20.4^{\circ}$ was clearly detected for the sample deposited under 0.5 mbar. It confirms that the $\mathrm{V}_{2} \mathrm{O}_{5}$ phase was present rather on the top of the thin film. Interestingly, the peak from a plane (200) $\left(15.5^{\circ}\right)$ was not detected, in contrast to the conventional XRD measurements. It may suggest a very specific arrangement of $\mathrm{V}_{2} \mathrm{O}_{5}$ crystallites. Additionally, the XRD patterns of samples deposited under 1 and 2 mbar consisted of peaks at $32.5^{\circ}$ and $48.5^{\circ}$ suggesting the coexistence of the $\mathrm{Bi}_{4} \mathrm{~V}_{2} \mathrm{O}_{11}$ (bismuth vanadate with Bi: $\mathrm{V}$ ratio 2:1), see Figure 3. Thus, the oxygen pressure in the deposition chamber affects the composition of resulting films. Such a conclusion was already formulated previously. However, the $\mathrm{Bi}_{4} \mathrm{~V}_{2} \mathrm{O}_{11}$ phase crystallized at low $\mathrm{O}_{2}$ pressure (unlike in this work), while the application of higher pressure in the deposition chamber led to the formation of pure $\mathrm{BiVO}_{4}$ [22]. The differences may result from (i) different substrates and (ii) different geometry of the deposition system. The crystallite size of the films was estimated on the basis of broadening of the XRD reflections. The crystallite size in (101) direction estimated from (101) reflection observed at $18.8^{\circ}$ was between 30 and $40 \mathrm{~nm}$. The largest crystallites were observed in the sample obtained at 0.1 mbar, whereas the crystallite size values in the other films were similar $(0.25-2 \mathrm{mbar})$. On the other hand, the reflection at $28.8^{\circ}$ corresponding to the (103) and (112) planes was narrower, the crystallite sizes were between 55 and $65 \mathrm{~nm}$. Additionally, in this case, low oxygen partial pressure lead to the formation of larger crystallites.

Samples have been investigated using the Raman spectroscopy technique. The films showed inhomogeneity and spectra vary depending on the measurement spot distance from the edge of the sample. Thus, the spectra were measured every $500 \mu \mathrm{m}$ from the edge that was the closest to the target during sputtering. Only in the case of the sample deposited under $0.1 \mathrm{mbar}$ pressure, the film is homogenous, and exemplary spectra are presented in Figure $4 \mathrm{a}$. However, besides bands coming from $\mathrm{BiVO}_{4}$, which are characterized by enhanced intensity due to the Raman resonance effect, the new low intensity bands are seen in the Raman spectra at $145,284,304,404,481,528$, and $995 \mathrm{~cm}^{-1}$. All these bands confirm the presence of $\mathrm{V}_{2} \mathrm{O}_{5}$. Interestingly, $\mathrm{V}_{2} \mathrm{O}_{5}$ was not registered on this sample using the XRD measurement, suggesting only short-range ordering in $\mathrm{V}_{2} \mathrm{O}_{5}$ or too small of an amount of it on the sample. Thus, the proposed method of deposition using PLD at certain $\mathrm{O}_{2}$ pressure leads to the formation of $\mathrm{BiVO}_{4} / \mathrm{V}_{2} \mathrm{O}_{5}$ heterojunction on the whole considered surface. The Raman spectra of the film deposited at higher oxygen pressure ( $0.25 \mathrm{mbar}$ ) are presented in Figure $4 \mathrm{~b}$. It can be concluded that $\mathrm{V}_{2} \mathrm{O}_{5}$ was not evenly distributed throughout the film. The highest intensity of the band coming from $\mathrm{V}_{2} \mathrm{O}_{5}\left(995 \mathrm{~cm}^{-1}\right)$ was detected 1000-2500 $\mu \mathrm{m}$ from the edge. In the further part of the layer, $\mathrm{V}_{2} \mathrm{O}_{5}$ does not occur and only bands originating from $\mathrm{BiVO}_{4}$ were detected. Samples obtained at pressure 0.5 mbar exhibited a similar distribution of $\mathrm{V}_{2} \mathrm{O}_{5}$ on the surface, see Figure 4c. The spectra recorded on the spots between 3000 and $6000 \mu \mathrm{m}$ from the sample edge were characteristic for the pure $\mathrm{BiVO}_{4}$ phase. Additionally, the spectrum measured at the opposite site of the layer exhibited a new feature (7000-8000 
$\mu \mathrm{m})$. The bands of $\mathrm{BiVO}_{4}$ merged and a new band at about $870 \mathrm{~cm}^{-1}$ appeared. In the case of the sample deposited under 1 mbar the effect of peaks merging was enhanced and visible over a larger area of the sample (5000-8000 $\mu \mathrm{m}$ ), while maintaining the presence of $\mathrm{V}_{2} \mathrm{O}_{5}$ on the opposite site of the layer (1000-3000 $\mu \mathrm{m})$, see Figure $4 \mathrm{~d}$. Notably, two phenomena do not coexist on any of the same spots of the sample and there is a "buffer layer" containing only $\mathrm{BiVO}_{4}(3000-5000 \mu \mathrm{m})$. In the case of the sample deposited at 2 mbar, the $\mathrm{V}_{2} \mathrm{O}_{5}$ was not detected, see Figure 4e. The spectra collected closest to the edge of the sample $(0-4000 \mu \mathrm{m})$ consisted of bands characteristic only of $\mathrm{BiVO}_{4}$. However, spectra change with distance from the sample edge and new bands at 247 and $567 \mathrm{~cm}^{-1}$ appear. Additionally, the two bands characteristic of V-O stretching vibration $\left(800-900 \mathrm{~cm}^{-1}\right.$ range) were clearly separated. Thus, depending on the pressure of oxygen in the deposition chamber, the composition of resulting films varied. For the low oxygen pressure, the effect of $\mathrm{V}_{2} \mathrm{O}_{5}$ formation was enhanced. $\mathrm{The}_{2} \mathrm{~V}_{2} \mathrm{O}_{5}$ peaks appeared closer to the sample edge that was closest to the target during deposition. In the case of higher pressures, the $\mathrm{V}_{2} \mathrm{O}_{5}$ disappeared and the opposite side of the sample contains a new phase that can be described as $\mathrm{Bi}$-rich bismuth vanadate- $-\mathrm{Bi}_{4} \mathrm{~V}_{2} \mathrm{O}_{11}$. The results obtained suggest that ablation performed using UV laser leads to the generation of plasma plume where $\mathrm{Bi}$ and $\mathrm{V}$ atoms/ions can be separated. The oxygen pressure may strongly affect $\mathrm{V}$ atoms/ions in plasma plume, but heavier $\mathrm{Bi}$ atoms/ions were not significantly slowed down due to interactions with oxygen molecules in the deposition chamber. As a result, depending on the oxygen pressure, the V-rich phase $\left(\mathrm{V}_{2} \mathrm{O}_{5}\right)$ can be formed on the one side of the sample, while the Bi-rich phase $\left(\mathrm{Bi}_{4} \mathrm{~V}_{2} \mathrm{O}_{11}\right)$ on the opposite. The effect of nonstoichiometric plasma plume propagation due to the differences of cations masses has been already reported for pulsed laser deposition of $\mathrm{LaAlO}_{3}$ and $\mathrm{LaGaO}_{3}[28,29]$. It was also reported on the basis on Langmuir probe measurements that lighter $\mathrm{Cu}_{+}$ions are more efficiently slowed down by collisions with oxygen molecules than heavier $\mathrm{Ba}^{+}$and $\mathrm{Y}^{+}$ions during deposition of $\mathrm{YBa}_{2} \mathrm{Cu}_{3} \mathrm{O}_{7}[30]$. EDX (energy-dispersive $\mathrm{X}$-ray spectroscopy) scans were performed in order to study the $\mathrm{Bi}: \mathrm{V}$ atomic ratio along the layer. The results for layers obtained under $0.1,0.5$, and 2 mbar are presented in Figure $4 f$. The spectra were measured point by point, approximately every $350 \mu \mathrm{m}$. The spread of results was relatively large, mainly due to irregularly occurring $\mathrm{V}_{2} \mathrm{O}_{5}$ crystallites, however the general relationship was clearly seen. The Bi:V ratio was close to one and more or less constant for films deposited at low pressures suggesting presence of $\mathrm{BiVO}_{4}$ phase. The Bi:V ratio lower than one confirmed the presence of the $\mathrm{V}_{2} \mathrm{O}_{5}$ phase. Higher pressure in the deposition chamber results in a compositional gradient across the layer and the Bi concentration increased (in comparison with $\mathrm{V}$ concentration) from the edge that was the closest to the target during deposition. EDX analysis confirmed the presence of Bi excess and formation of $\mathrm{Bi}_{4} \mathrm{~V}_{2} \mathrm{O}_{11}$ for the films deposited under 2 mbar. The $\mathrm{Bi}: \mathrm{V}$ ratio was not equal to 2, because two phases are present $\left(\mathrm{BiVO}_{4}\right.$ and $\left.\mathrm{Bi}_{4} \mathrm{~V}_{2} \mathrm{O}_{11}\right)$. The results obtained support the hypothesis that $\mathrm{Bi}$ and $\mathrm{V}$ atoms and ions in plasma plume can be separated due to the interactions or reaction with $\mathrm{O}_{2}$ gas, what affects the spatial distribution of elements on the deposited films.

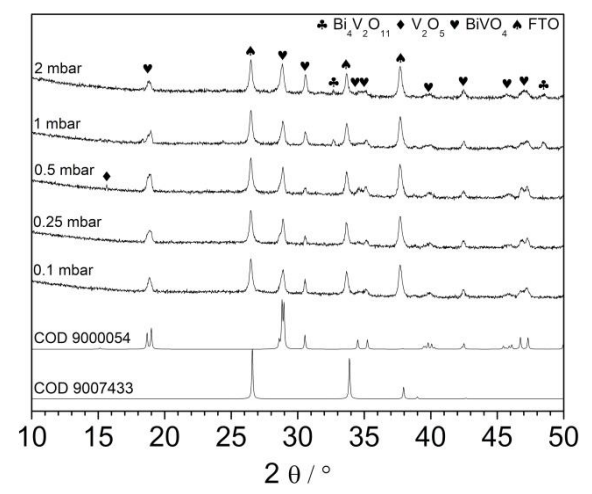

Figure 3. The comparison of XRD patterns recorded for $\mathrm{FTO} / \mathrm{BiVO}_{4}$ samples deposited at various oxygen pressures. $\mathrm{B}$ and $\mathrm{V}$ symbols denote $\mathrm{Bi}_{4} \mathrm{~V}_{2} \mathrm{O}_{11}$ and $\mathrm{V}_{2} \mathrm{O}_{5}$, respectively. COD 9000054- $\mathrm{BiVO}_{4}$ and $\mathrm{COD} 9007433-\mathrm{SnO}_{2}$. 
a)

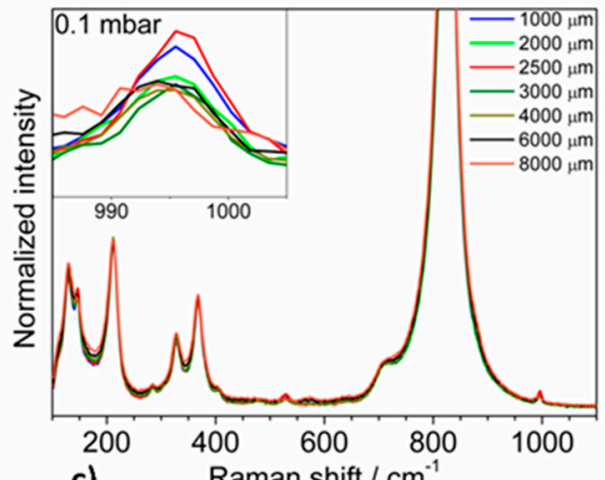

c)

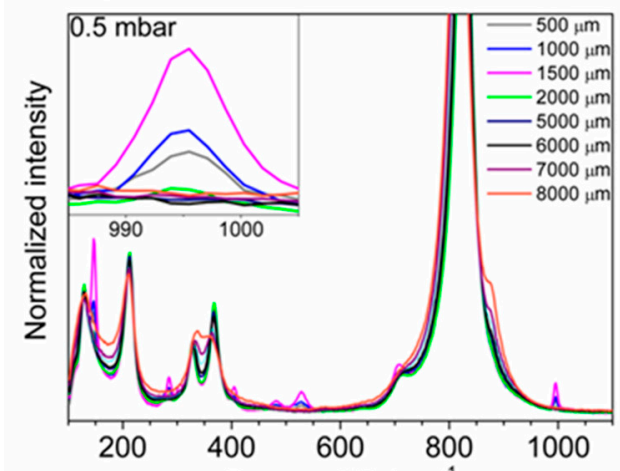

e) Raman shift $/ \mathrm{cm}^{-1}$

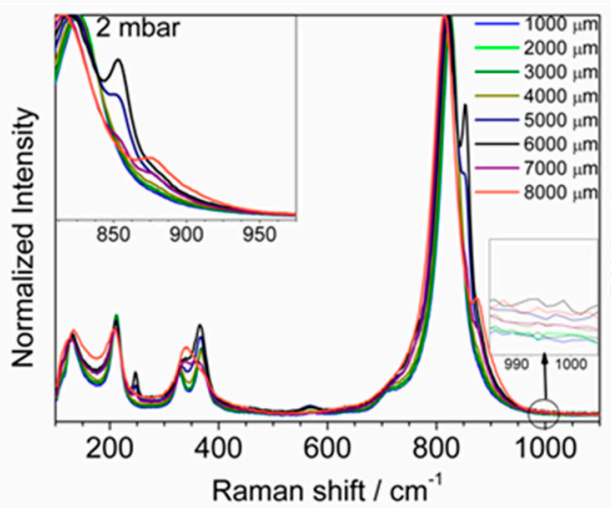

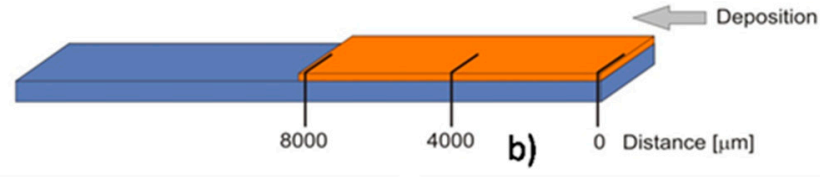

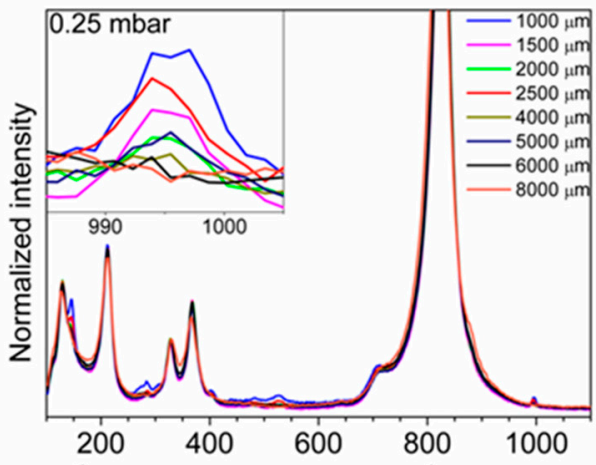

d)
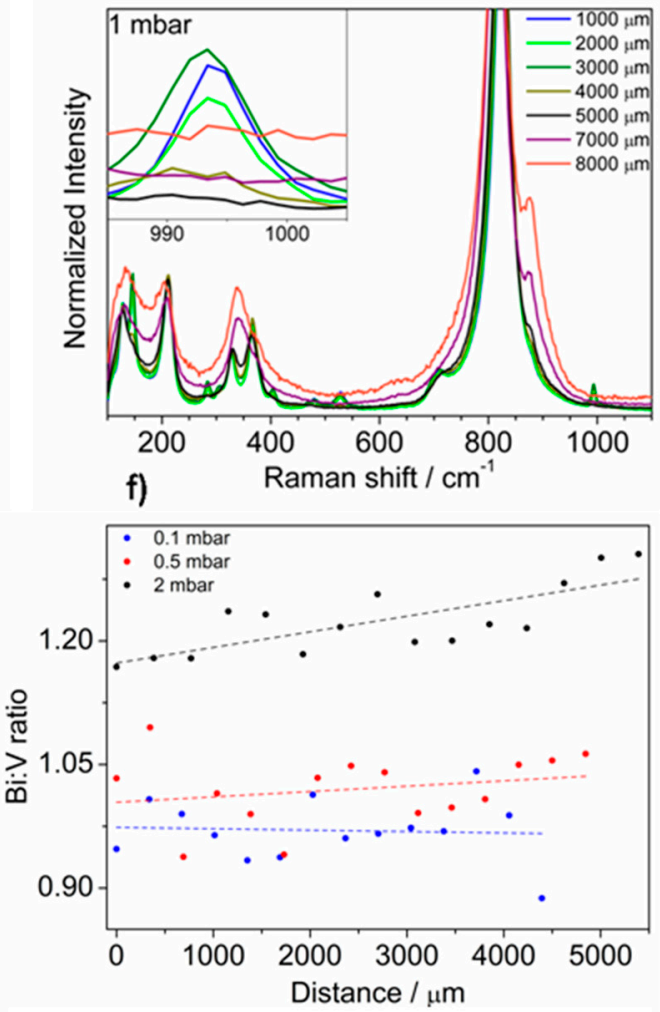

Figure 4. (a-e) Raman spectra of $\mathrm{FTO} / \mathrm{BiVO}_{4}$ samples deposited under different pressures measured at different spots and (f) Bi: $\mathrm{V}$ ratio estimated on the basis of EDX measurements.

The morphology and composition of the spent target after deposition was investigated. The SEM micrograph of a target's area exposed to laser pulses is presented in Figure 5a. Generally, the area is rather flat and $\mathrm{BiVO}_{4}$ particles cannot be distinguished, however some pinholes were formed. Raman spectrum of the area is characteristic of $\mathrm{BiVO}_{4}$ and $\mathrm{V}$ and Bi rich phases were not detected, see the inset of Figure 5a. It suggests that the ablation causes a formation of a plasma with a rather stoichiometric $(\mathrm{Bi}: \mathrm{V})$ composition. The pressure did not affect the ablation thresholds of $\mathrm{V}$ and $\mathrm{Bi}$ independently. The area of a target that was uncovered during deposition but not exposed to the laser pulses was also investigated. The SEM image is presented in Figure $5 \mathrm{~b}$. The surface was covered by a new phase, which was not observed before the PLD procedure (compared with Figure 1c). According to the Raman spectroscopy measurements, small particles contained the $\mathrm{V}_{2} \mathrm{O}_{5}$ phase as is shown in the inset of Figure $5 b$, however the main signal comes from the underlying $\mathrm{BiVO}_{4}$. Thus, vanadium was 
significantly affected by the oxygen gas in the chamber and it led to redeposition only of $\mathrm{V}_{2} \mathrm{O}_{5}$ on the target. Bi-rich phases were not detected there. Thus, it is very likely that the $\mathrm{Bi} / \mathrm{V}$ separation occurs after ablation, during interactions with oxygen molecules with ions characterized by different masses.

a)

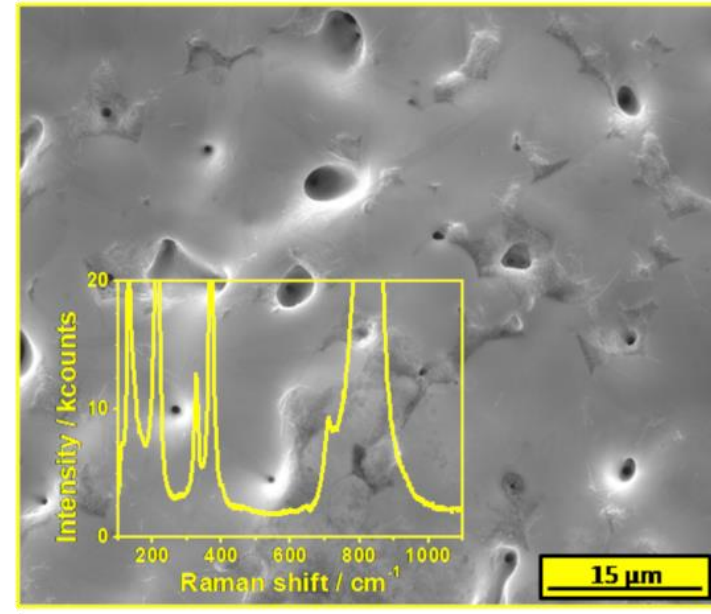

b)

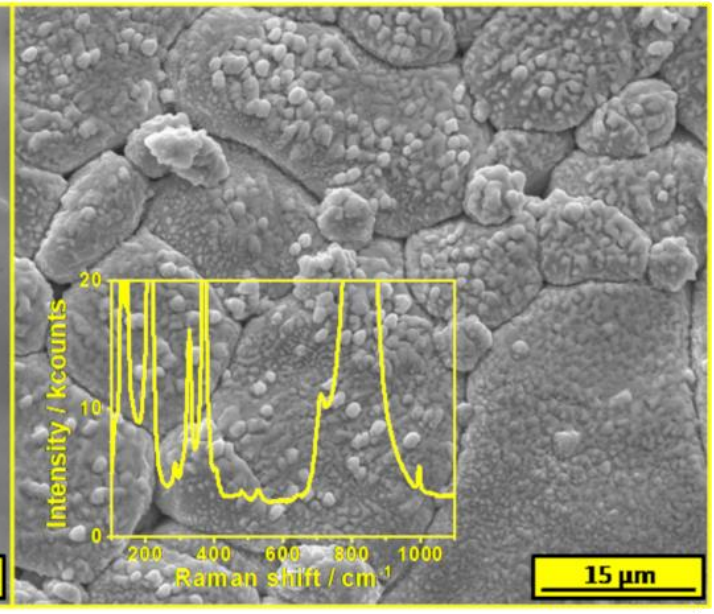

Figure 5. The SEM images and Raman spectra of a $\mathrm{BiVO}_{4}$ target after pulsed laser deposition. (a) The area exposed to the laser pulses and (b) the area not subjected to the ablation.

The morphology of layers was investigated using scanning electron microscopy. SEM images were recorded at three different spots for each sample, as is shown in Figure 6. The sample deposited at the lowest pressure of $\mathrm{O}_{2}$ was quite homogenous, which was consistent with the Raman spectroscopy measurements. Films were built by round-shaped grains with different diameters from about 100 to $200 \mathrm{~nm}$. Inclusion of another phase, probably $\mathrm{V}_{2} \mathrm{O}_{5}$, was also visible. The higher pressure during deposition affected the amount of the $\mathrm{V}_{2} \mathrm{O}_{5}$ phase on the film surface. Layers deposited under $0.5 \mathrm{mbar}$ were built by clearly bigger particles of $\mathrm{BiVO}_{4}$. An additional phase of $\mathrm{V}_{2} \mathrm{O}_{5}$ on the top of $\mathrm{BiVO}_{4} \mathrm{Was}$ observed in a form of protruding longitudinal crystallites (confirmed by EDX analysis, see Figure S4), only near the edge of the sample that was the closest to the target. The cross-sectional micrograph of area rich in specific structures of the sample deposited under 0.5 mbar is presented in Figure S5. Although the $\mathrm{V}_{2} \mathrm{O}_{5}$ phase was also present on the surface of the samples deposited under 0.1 and 0.25 mbar (which was confirmed by Raman spectroscopy), the XRD technique enabled detection of it only in the case of the sample deposited under 0.5 mbar on which crystallites were formed. The effect of Bi to the $\mathrm{V}$ ratio on the morphology was visible on SEM images of the samples deposited under 1 and 2 mbar. The higher concentration of bismuth made the crystallite edges sharper, while a lower Bi concentration area was characterized by rather round-shape grains. It should be noticed that the separated phase of $\mathrm{Bi}_{4} \mathrm{~V}_{2} \mathrm{O}_{11}$ could not be distinguished on the SEM micrographs. The SEM image of the larger area of the sample deposited under 2 mbar was added to the Supplementary Information in order to prove that there are no separated crystallites of $\mathrm{Bi}_{4} \mathrm{~V}_{2} \mathrm{O}_{11}$, see Figure S6. It is very likely that the bulk $\mathrm{Bi}_{4} \mathrm{~V}_{2} \mathrm{O}_{11} @ \mathrm{BiVO}_{4}$ heterojunction was formed, as opposed to $\mathrm{BiVO}_{4} / \mathrm{V}_{2} \mathrm{O}_{5}$. It has been already shown previously that $\mathrm{BiVO}_{4}$ and $\mathrm{Bi}_{4} \mathrm{~V}_{2} \mathrm{O}_{11}$ tend to form structures in which the components are indistinguishable (by SEM technique) due to the formation of nanosized interfacial contact [31]. Additionally, AFM measurements were performed In order to characterize the topography of the deposited films. The results are presented in Figure S7. The root mean square roughness of the sample deposited under 0.1 mbar was the lowest $(32 \mathrm{~nm})$, while the rest of the samples were characterized by rms equal to $38 \mathrm{~nm}$. 

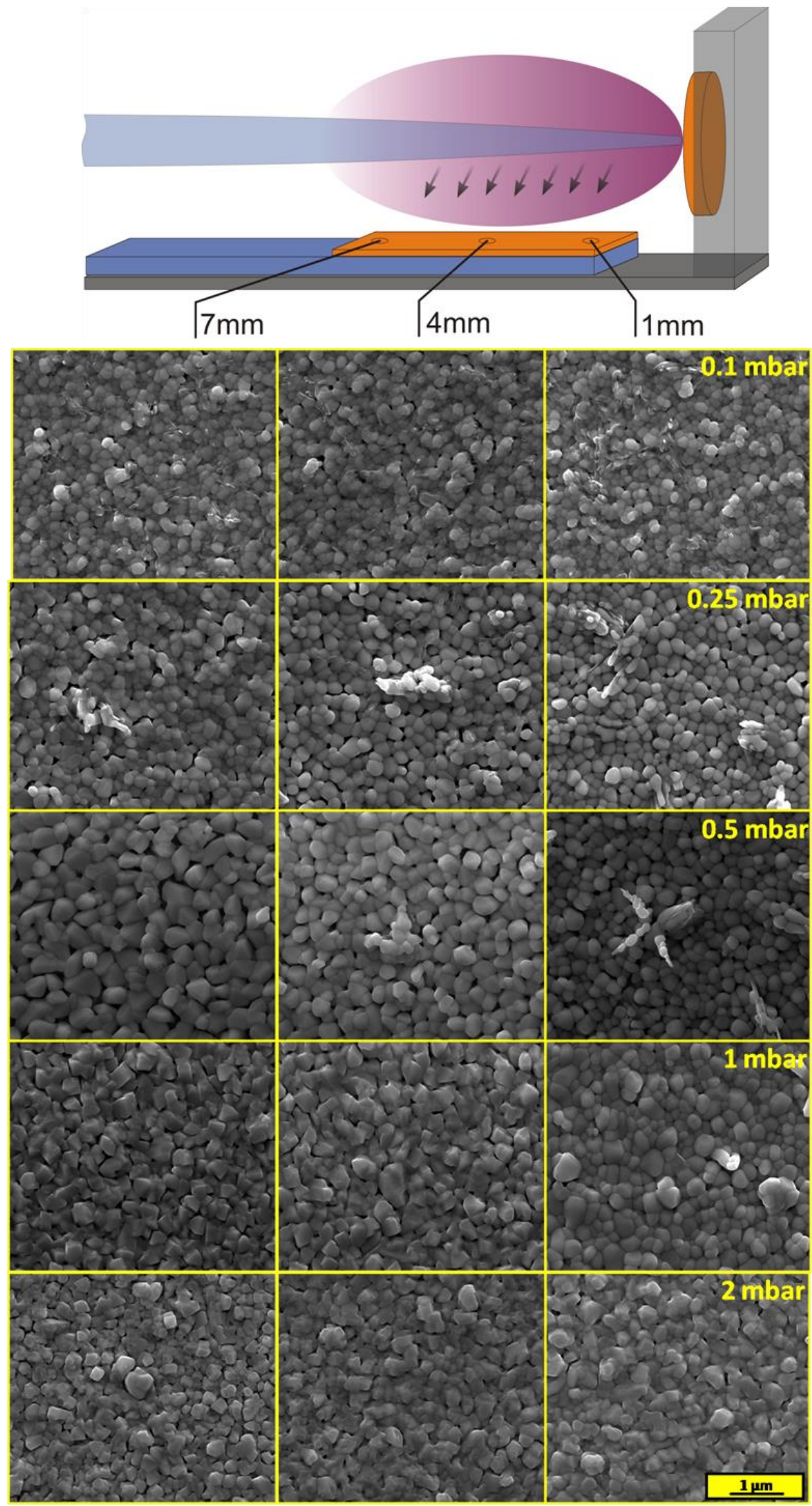

Figure 6. SEM images taken at different spots of the samples deposited under $0.1,0.25,0.5,1$, and 2 mbar. 
The plasma plume formed during deposition was also characterized using LIBS (laser-induced breakdown spectroscopy). The investigated radiation spectra contained clear atomic lines corresponding to oxygen, bismuth, and vanadium as well as continuous molecular spectra, which identification would require higher spectral resolution. Most probably these lines may correspond to oxygen and ozone molecules as well as bismuth and vanadium oxides or dimmers. The LIBS spectra recorded at different distances from the target during $\mathrm{BiVO}_{4}$ deposition $(\mathrm{p}=2 \mathrm{mbar}$ ) are presented in Figure 7 . The analyzed lines are marked in the upper part of the picture.

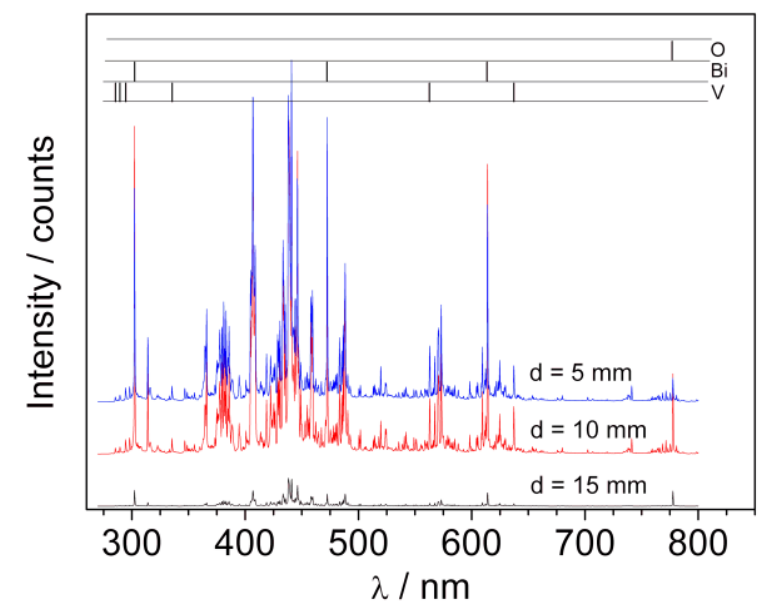

Figure 7. LIB spectra recorded during pulsed laser deposition process at different distances from target and oxygen pressure 2 mbar.

In the case of the signal coming from oxygen, there is a high intensity triplet $\mathrm{O}(777)$-777.194, 777.417 , and $777.539 \mathrm{~nm}$ (here seen as one line around $777 \mathrm{~nm}$ ). The radiation results from optical transition $3 s^{5} S^{0}{ }_{2} \rightarrow 3 p^{5} P_{12}$. The energy of the higher transition level is around $10.74 \mathrm{eV}$ and was not detected here. The bismuth lines observed in the spectra correspond to the transition from high energy levels $7 s^{2,4} P, 8 s^{2} P$, and $6 d^{2} D$ (with energies in the range of $4-6 \mathrm{eV}$ ) to metastable $6 p^{32} D^{0} 3 / 2,5 / 2$ and $6 p^{32} P^{0} 1 / 2,3 / 2$. Lines corresponding to optical resonance transition (to ground electronic state $6 p^{34} S^{0}{ }_{3 / 2}$ ) were not observed, which can be explained by full absorption in plasma volume. A radiation from vanadium atoms contains a large amount of spectral lines, which constitute the main part of the observed spectra. Energies of excited levels $\left(3 d^{3} 4 s 4 p^{4} D^{0}, 3 d^{4} 4 p^{4} P\right.$, and $\left.3 d^{4} 4 p^{4} F\right)$ for observed lines were of the order $4 \div 5.3 \mathrm{eV}$. The observed transitions end in the ground electronic state $\left(3 d^{3} 4 s^{2}{ }^{4} F\right)$ or metastable levels $\left(3 d^{4} 4 s^{6} \mathrm{D}\right.$ and $\left.3 d^{3} 4 s^{2}{ }^{4} P\right)$. It should be underlined that in contrast to the bismuth spectra, in the case of vanadium the electrons fell to metastable as well as ground states. This means that the resonance lines of vanadium were reabsorbed in the plasma less than those of bismuth. It may point to the fact that there were significantly fewer atoms of vanadium than bismuth in the investigated plasma. Since Bi and V ablate from the target in a stoichiometric way, the results obtained indicate that propagation of vanadium in the deposition chamber filled with oxygen was therefore difficult.

The intensities of the selected lines were plotted versus the distance from the $\mathrm{BiVO}_{4}$ target and the resulting graphs are presented in Figure 8. Measurements were performed under 0.2 and $2 \mathrm{mbar}$ oxygen pressure. The dependence of intensities on a distance for low oxygen pressure was very similar for all observed vanadium lines, i.e., the intensities grew for a distance $\mathrm{L}<10 \mathrm{~mm}$ and suddenly dropped later (Figure 8a). Similar dependences were observed in the case of bismuth (Figure 8c). As oxygen pressure grows the dependence changes. The maximum of intensities was shifted to lower $\mathrm{L}$ values, they grew for $\mathrm{L}<5 \mathrm{~mm}$ and fell rapidly later, see Figure $8 \mathrm{~b}$, d. The dependences differed significantly in the case of oxygen both in the case of lower and higher oxygen pressure, see Figure 8e,f. Oxygen lines may originate from the oxygen ablated from the target. However, it is very likely that 
signals at about $777 \mathrm{~nm}$ originate from oxygen (ambient gas) species excited during interaction with plasma components.

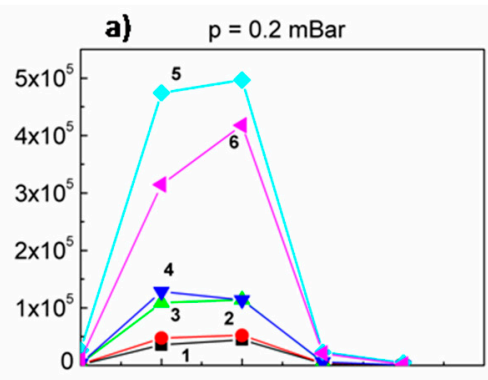

c)

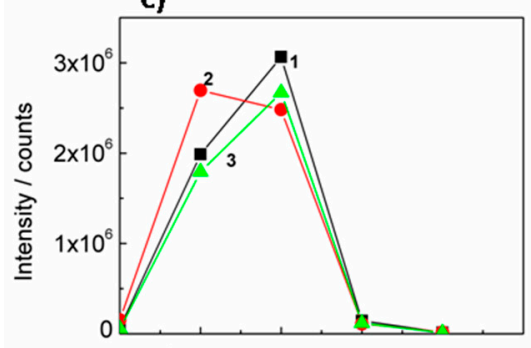

e)

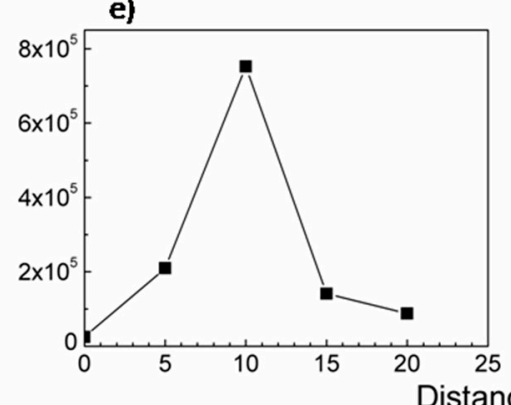

b) $\quad \mathrm{p}=2 \mathrm{mBar}$

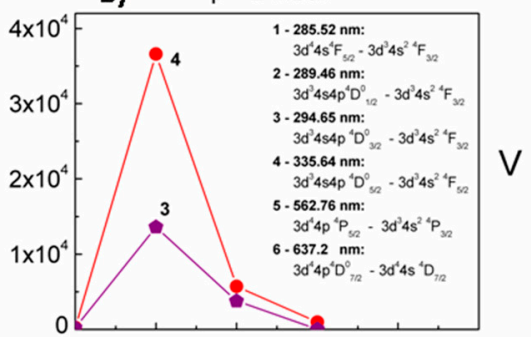

d)

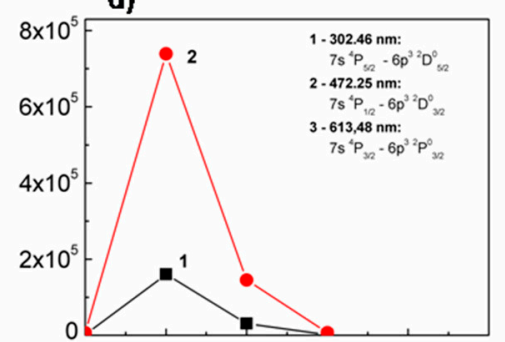

f)

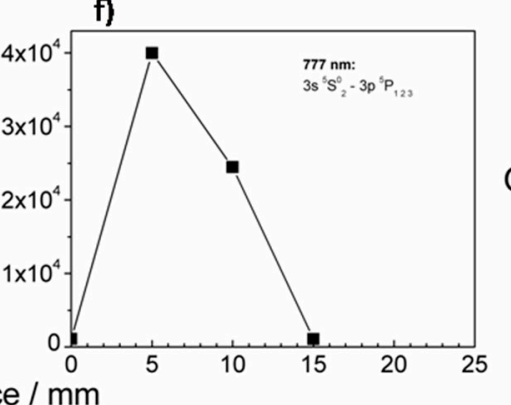

Figure 8. Line intensities as a function of distance from target for transitions in vanadium $(\mathbf{a}, \mathbf{b})$ $1-3 d^{4} 4 s^{4} F_{5 / 2}-3 d^{3} 4 s^{2}{ }^{4} F_{3 / 2}, 2-3 d^{3} 4 s 4 p^{4} D^{0}{ }_{1 / 2}-3 d^{3} 4 s^{2}{ }^{4} F_{3 / 2}, 3-3 d^{3} 4 s 4 p^{4} D^{0}{ }_{3 / 2}-3 d^{3} 4 s^{2}{ }^{4} F_{3 / 2}, 4-3 d^{3} 4 s 4 p$ ${ }^{4} D^{0}{ }_{5 / 2}-3 d^{3} 4 s^{2}{ }^{4} F_{5 / 2}, 5-3 d^{4} 4 p^{4} P_{5 / 2}-3 d^{3} 4 s^{2}{ }^{4} P_{3 / 2}$, and $6-3 d^{4} 4 p^{4} D^{0} 7 / 2-3 d^{4} 4 s^{4} D_{7 / 2}$, for atomic bismuth (c,d) for transitions $1-7 s{ }^{4} P_{5 / 2}-6 p^{32} D^{0} 5 / 2,2-7 s{ }^{4} P_{1 / 2}-6 p^{32} D^{0}{ }_{3 / 2}$, and $3-7 s{ }^{4} P_{3 / 2}-6 p^{32} P^{0}$, , and for atomic oxygen (e,f) for triplet $3 s^{5} S^{0}{ }_{2} \rightarrow 3 p^{5} P_{123}$.

During the laser ablation in the presence of ambient gas, the ablated species were decelerated and attenuated due to collision with gas molecules. These processes resulted in plasma confinement and generation of shock waves at the plasma propagation front, where chemical reactions of ablated materials with oxygen molecules were intensified. Higher ambient gas pressure results in the compression of plasma and shifting of the plasma front into the proximity of target. It was observed in the experiment as a change of spectral lines intensities versus the distance from a target measured for two different pressure values (Figure 8). For a higher pressure the maximum intensity of excited oxygen shifted towards the target, which means that the zone of intensive collision excitation also shift. The decrease of intensity beyond the maximum indicates a collision recombination. The attenuation of the emission with an increase of distance from the target may result from non-radiative processes (including quenching and energy transfer processes) and adiabatic expansion of the plasma. However the kinetic energy of all species was still sufficient to reach the substrate and form thin film. In our experiment, we observed the presence of the $\mathrm{V}_{2} \mathrm{O}_{5}$ phase, in a form of irregularly occurring crystallites, that for higher oxygen pressure was shifted towards the target. This was in an agreement with the spectroscopic observation of plasma properties. It should be also noted that the increase of ambient gas pressure resulted in the decrease of plasma plume species velocity, which can influence the morphology of the deposited film. For a higher pressure and distances from the target, where relaxation processes in 
plasma could be observed, the thin film was more homogenous, which was confirmed by SEM images (see Figure S6). The excess of vanadium in one part of the sample caused its deficiency in the area of the covered substrate, which was farther from the target. The effect of this phenomenon was a higher $\mathrm{Bi}: \mathrm{V}$ ratio and possibility of $\mathrm{Bi}_{4} \mathrm{~V}_{2} \mathrm{O}_{11}$ crystallization and formation of the $\mathrm{BiVO}_{4} / \mathrm{Bi}_{4} \mathrm{~V}_{2} \mathrm{O}_{11}$ system.

The most promising application of thin layers of $\mathrm{BiVO}_{4}$ is photoelectrochemical water splitting. Thus, the obtained films were tested as photoanodes for water photooxidation under simulated solar light exposure. As it is shown in Figure 9a, the shape of linear sweep voltammograms was similar for each sample and the presence of heterojunctions $\left(\mathrm{BiVO}_{4} / \mathrm{V}_{2} \mathrm{O}_{5}\right.$ and $\left.\mathrm{Bi}_{4} \mathrm{~V}_{2} \mathrm{O}_{11} @ \mathrm{BiVO}_{4}\right)$ did not clearly affect it. The curves were characteristic of n-type semiconductor electrodes under illumination [32]. The lowest photoactivity was observed for samples deposited under 0.1 mbar. Generally, the formation of $\mathrm{BiVO}_{4} / \mathrm{V}_{2} \mathrm{O}_{5}$ heterojunction can enhance the efficiency of photocurrent generation for both, layered and bulk types of junctions [10]. However, the geometry of the system significantly affects its photoactivity [33] due to the relative positions of conduction bands [34]. The photoexcited electrons can be trapped due to the potential barrier if the $\mathrm{V}_{2} \mathrm{O}_{5}$ is mainly on the top of the electrode, as it is in the present work. Thus, $\mathrm{FTO} / \mathrm{BiVO}_{4}$ photoanode deposited under 0.1 mbar was immersed in $1 \mathrm{M} \mathrm{NaOH}$ in order to facilitate selective dissolution of $\mathrm{V}_{2} \mathrm{O}_{5}$. This is a procedure commonly used during some methods of $\mathrm{BiVO}_{4}$ synthesis that uses the excess of the V-containing precursor [35]. The comparison of the LSV (linear sweep voltammetry) curves recorded before and after soaking in $\mathrm{NaOH}$ solution is presented in Figure 9b. Since the enhancement of photocurrent was achieved, the presented results confirmed the negative influence of $\mathrm{V}_{2} \mathrm{O}_{5}$ on the surface on $\mathrm{BiVO}_{4}$. If $\mathrm{BiVO}_{4}$ and $\mathrm{V}_{2} \mathrm{O}_{5}$ did not form a heterojunction, an increase in photocurrent in comparison with $\mathrm{BiVO}_{4}$ would be expected due to the narrower energy band gap of vanadium pentaoxide. In the case of the sample deposited under $0.5 \mathrm{mbar}$, most of the sample surface consists of bare $\mathrm{BiVO}_{4}$. The enhancement of the photocurrent, in comparison with samples deposited under lower pressures, results from the lack of adverse $\mathrm{BiVO}_{4} / \mathrm{V}_{2} \mathrm{O}_{5}$ junction presence on a larger area of the film. The highest photocurrent of water oxidation was observed for the sample deposited under $1 \mathrm{mbar}$, however the system was very complex due to the presence of three phases: $\mathrm{BiVO}_{4}, \mathrm{~V}_{2} \mathrm{O}_{5}$, and $\mathrm{Bi}_{4} \mathrm{~V}_{2} \mathrm{O}_{11}$. A very similar result was achieved for the sample that consisted of the $\mathrm{Bi}_{4} \mathrm{~V}_{2} \mathrm{O}_{11} @ \mathrm{BiVO}_{4}$ heterojunction (deposited under 2 mbar). Notably, pristine $\mathrm{FTO} / \mathrm{BiVO}_{4}$ photoanodes (prepared under 0.1 mbar with removed $\mathrm{V}_{2} \mathrm{O}_{5}$ ) generated a lower photocurrent than photoanodes consisting of the $\mathrm{BiVO}_{4} @ \mathrm{Bi}_{4} \mathrm{~V}_{2} \mathrm{O}_{11}$ heterojunction. It has been already reported that such a system positively affects the photoelectroactivity in comparison to bare $\mathrm{BiVO}_{4}[31,36]$. The geometry of the system should be taken into account, as it was discussed for the $\mathrm{BiVO}_{4} / \mathrm{V}_{2} \mathrm{O}_{5}$ photoanode. However, the crystallites of $\mathrm{Bi}_{4} \mathrm{~V}_{2} \mathrm{O}_{11}$ cannot be simply distinguished on the SEM images, thus it is very likely that bulk heterojunction was obtained. Since the pulsed laser deposited films of $\mathrm{Bi}_{4} \mathrm{~V}_{2} \mathrm{O}_{11}$ acts as a rather poor photoanode i.a. due to the low absorption coefficient [37], in the case of the formation of $\mathrm{BiVO}_{4}$ and $\mathrm{Bi}_{4} \mathrm{~V}_{2} \mathrm{O}_{11}$ separated phases, the enhancement would not be expected. Thus, the formation of heterojunction is the main reason of photocurrent improvement. The formation of heterojunction leads to the inhibition of bulk electron/hole recombination [38]. Generally, the presence of type-II heterojunction enhances bulk $\mathrm{e}^{-} / \mathrm{h}^{+}$separation efficiency due to the internal electric field generated on the phases interface, e.g., $\mathrm{BiVO}_{4} / \mathrm{Bi}_{4} \mathrm{~V}_{2} \mathrm{O}_{11}$ [36]. The enhancement of photoactivity in the case of the $\mathrm{Bi}_{4} \mathrm{~V}_{2} \mathrm{O}_{11}$-containing photoanode may be also related to improved charge transfer resistance through the photoactive film in comparison with pristine $\mathrm{BiVO}_{4}$ as it was reported previously [22]. It is not expected that improvement is related to better light absorption because the energy band gaps of $\mathrm{BiVO}_{4}$ and $\mathrm{Bi}_{4} \mathrm{~V}_{2} \mathrm{O}_{11}$ are comparable [39]. Since the morphology, thickness, and roughness of the samples were comparable, we claimed that differences in the photocurrent of water oxidation were related mainly to the composition of the samples. The recorded photocurrent seems to be lower in comparison with other pulsed laser deposited $\mathrm{BiVO}_{4}$-based photoanodes [22,40,41]. However it should be noticed that in the case of the presented results the photocurrent was related to water photooxidation and oxygen evolution, not oxidation of the consumable hole scavenger. On the other hand, results were 
comparable with the photocurrent of water oxidation that was achieved for unmodified $\mathrm{BiVO}_{4}$ films with underlying hole-blocking layer sputtered using the PLD method [42].
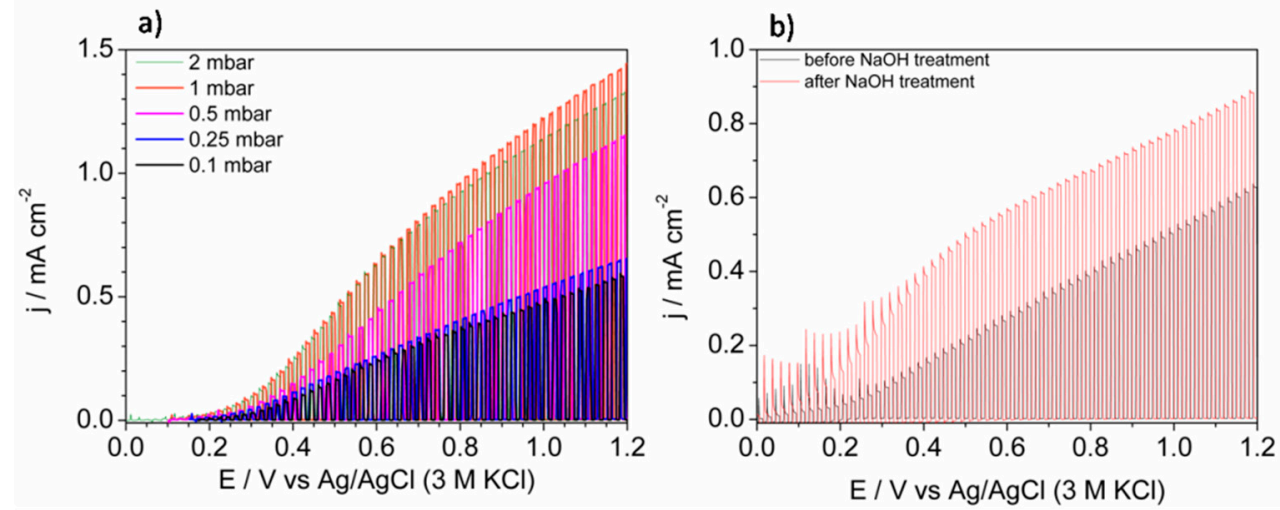

Figure 9. (a) The comparison of LSV curves recorded in $0.2 \mathrm{M} \mathrm{K}_{2} \mathrm{SO}_{4}$ under intermittent AM 1.5 illumination for $\mathrm{FTO} / \mathrm{BiVO}_{4}$ photoanodes deposited under various oxygen pressures. (b) The comparison of LSV curves recorded for $\mathrm{FTO} / \mathrm{BiVO}_{4}$ photoanodes ( 0.1 mbar) before and after $\mathrm{NaOH}$ treatment. Scan rate was $20 \mathrm{mV} \mathrm{s}^{-1}$.

\section{Summary}

In summary, we successfully performed the deposition of photoactive films on FTO substrates from the $\mathrm{BiVO}_{4}$ target using the pulsed laser deposition technique. The influence of oxygen pressure in a deposition chamber on composition, morphology, and photoelectrochemical properties of layers was studied. It was shown that $\mathrm{BiVO}_{4} / \mathrm{V}_{2} \mathrm{O}_{5}$ heterojunction could be formed in the case of lower $\mathrm{O}_{2}$ pressures $(0.1,0.250 .5$, and $1 \mathrm{mbar})$, while $\mathrm{Bi}_{4} \mathrm{~V}_{2} \mathrm{O}_{11} @ \mathrm{BiVO}_{4}$ bulk heterojunction was formed in the case of higher oxygen pressures ( 1 and 2 mbar). Comparing the results of XRD, Raman spectroscopy, EDX, and SEM, it could be concluded that in the case of low pressures, the distribution of the additional $\mathrm{V}_{2} \mathrm{O}_{5}$ phase was quite homogeneous, and for moderate pressures separate $\mathrm{V}_{2} \mathrm{O}_{5}$ structures were formed in some areas of the investigated films. In the case of the films deposited at high oxygen pressures, the morphology of the area that contained $\mathrm{BiVO}_{4} @ \mathrm{Bi}_{4} \mathrm{~V}_{2} \mathrm{O}_{11}$ differed from bare $\mathrm{BiVO}_{4}$, however, the new, separated phase could not be distinguish on the SEM images, suggesting the formation of a well distributed $\mathrm{BiVO}_{4} @ \mathrm{Bi}_{4} \mathrm{~V}_{2} \mathrm{O}_{11}$ heterojunction. The formation of new phases $\left(\mathrm{V}_{2} \mathrm{O}_{5}\right.$ and $\left.\mathrm{Bi}_{4} \mathrm{~V}_{2} \mathrm{O}_{11}\right)$ was strictly related to the pressure and the location of the investigated area on the sample. According to our results, it could be concluded that the stoichiometry and composition could be tuned by changing the oxygen pressure in the deposition chamber and the distance of the substrate from the target during sputtering. The characterization of the target performed after exposure to laser pulses showed that $\mathrm{Bi}$ and $\mathrm{V}$ ablate from the target in a stoichiometric way, thus the separation of Bi and V atoms occurred in a plasma plume due to interactions with oxygen molecules. As a result, there were areas on the deposited samples that were enriched in $\mathrm{V}\left(\mathrm{BiVO}_{4} / \mathrm{V}_{2} \mathrm{O}_{5}\right)$ and $\mathrm{Bi}\left(\mathrm{Bi}_{4} \mathrm{~V}_{2} \mathrm{O}_{11} @ \mathrm{BiVO}_{4}\right)$, depending on the distance from the target during the deposition. According to the LIBS measurements, there were transition lines characteristic for vanadium, bismuth, and oxygen. Unlike bismuth transitions, vanadium-specific transition lines end in a ground-state, suggesting that there were fewer $\mathrm{V}$ atoms in plasma plume at measurements spots. The photoanodes prepared under different oxygen pressures exhibited different photoelectrochemical properties. In the case of $\mathrm{FTO} / \mathrm{BiVO}_{4} / \mathrm{V}_{2} \mathrm{O}_{5}$ films deposited under low pressure, photoactivity measured as a photocurrent of water oxidation was worse in comparison to $\mathrm{FTO} / \mathrm{Bi}_{4} \mathrm{~V}_{2} \mathrm{O}_{11} @ \mathrm{BiVO}_{4}$ photoanodes obtained under higher $\mathrm{O}_{2}$ pressure. The presence of the $\mathrm{Bi}_{4} \mathrm{~V}_{2} \mathrm{O}_{11} @ \mathrm{BiVO}_{4}$ heterojunction positively affected the photocurrent in comparison with pristine $\mathrm{BiVO}_{4}$ photoanodes as well. 


\section{Conclusions}

The formation of $\mathrm{V}_{2} \mathrm{O}_{5}$ and $\mathrm{Bi}_{4} \mathrm{~V}_{2} \mathrm{O}_{11}$ phases during $\mathrm{BiVO}_{4}$ deposition using the pulsed laser deposition technique was strictly related to the oxygen pressure and the location of the investigated area on the sample. According to our results, it could be concluded that the stoichiometry and composition could be tuned by changing the oxygen pressure in the deposition chamber and the distance of the substrate from the target during sputtering. The problem with non-stoichiometric deposition of multielemental materials could be related to the different scattering and interaction of ablated ions with gas molecules present in the chamber. Among the tested electrodes, the highest photoactivity was achieved for the samples that consisted of the $\mathrm{BiVO}_{4} / \mathrm{Bi}_{4} \mathrm{~V}_{2} \mathrm{O}_{11}$ bulk heterojunction.

Supplementary Materials: The following are available online at http://www.mdpi.com/1996-1944/13/6/1360/s1, Figure S1: The self-made system for pulsed laser deposition. Figure S2: The exemplary cross-sectional SEM micrograph of the sample deposited under 0.1 mbar. Figure S3: The small-angle XRD patterns of the samples deposited under 0.1, 0.5, and 2 mbar. Figure S4: The EDX analysis of the $\mathrm{V}_{2} \mathrm{O}_{5}$ crystals formed on the $\mathrm{BiVO}_{4}$ film deposited under 0.5 mbar. Figure S5: The cross-sectional SEM micrograph of the area rich in longitudinal crystallites. Figure S6: The SEM image of the sample deposited under 2 mbar. Figure S7: AFM topography of films deposited under $0.1,0.25,0.5,1$, and 2 mbar. Table S1: The variation of the film thickness depending on the oxygen pressure and the distance from the target.

Author Contributions: K.T.: Conceptualization, Investigation, Writing-original draft, Writing-review and editing. M.S. (Mariusz Szkoda): Validation, M.G.: Investigation. J.K.: Investigation. A.C.: Writing一original draft. G.M.G.: Formal analysis. M.S. (Mirosław Sawczak): Investigation, Methodology, Visualization. All authors have read and agreed to the published version of the manuscript.

Funding: Author K.T. gratefully acknowledges financial support from the Statutory Funds of Gdańsk University of Technology (DS 033209). This work is financially supported by The National Centre for Research and Development via grant no LIDER/15/0088/L-10/18/NCBR/2019 (Integrated prototype of a photo-supercapacitor for energy storage obtained as a result of solar radiation conversion).

Conflicts of Interest: The authors declare no conflict of interest.

\section{References}

1. Tayebi, M.; Lee, B. Recent advances in $\mathrm{BiVO}_{4}$ semiconductor materials for hydrogen production using photoelectrochemical water splitting. Renew. Sustain. Energy Rev. 2019, 111, 332-343. [CrossRef]

2. Stoughton, S.; Showak, M.; Mao, Q.; Koirala, P.; Hillsberry, D.A.; Sallis, S.; Kourkoutis, L.F.; Nguyen, K.; Piper, L.F.J.; Tenne, D.A.; et al. Adsorption-controlled growth of $\mathrm{BiVO}_{4}$ by molecular-beam epitaxy. APL Mater. 2013, 1, 42112. [CrossRef]

3. Zhao, Z.; Li, Z.; Zou, Z. Electronic structure and optical properties of monoclinic clinobisvanite $\mathrm{BiVO}_{4}$. Phys. Chem. Chem. Phys. 2011, 13, 4746-4753. [CrossRef]

4. Li, T.; He, J.; Peña, B.; Berlinguette, C.P. Curing $B i V O_{4}$ Photoanodes with Ultraviolet Light Enhances Photoelectrocatalysis. Angew. Chem. 2016, 55, 1769-1772. [CrossRef] [PubMed]

5. Prévot, M.S.; Sivula, K. Photoelectrochemical Tandem Cells for Solar Water Splitting. J. Phys. Chem. C 2013, 117, 17879-17893. [CrossRef]

6. Tan, H.L.; Amal, R.; Ng, Y.H. Alternative strategies in improving the photocatalytic and photoelectrochemical activities of visible light-driven BiVO4: A review. J. Mater. Chem. A 2017, 5, 16498-16521. [CrossRef]

7. Thalluri, S.M.; Hernández, S.; Bensaid, S.; Saracco, G.; Russo, N. Green-synthesized W- and Mo-doped $\mathrm{BiVO}_{4}$ oriented along the $\{040\}$ facet with enhanced activity for the sun-driven water oxidation. Appl. Catal. B Environ. 2016, 180, 630-636. [CrossRef]

8. Kim, J.H.; Lee, J.S. BiVO 4 -Based Heterostructured Photocatalysts for Solar Water Splitting: A Review. Energy Environ. Focus 2014, 3, 339-353. [CrossRef]

9. Kim, K.; Nam, S.K.; Park, J.H.; Moon, J.H. Growth of $\mathrm{BiVO}_{4}$ nanoparticles on a $\mathrm{WO}_{3}$ porous scaffold: Improved water-splitting by high band-edge light harvesting. J. Mater. Chem. A 2019, 7, 4480-4485. [CrossRef]

10. Oliveira, A.T.; Rodriguez, M.; Andrade, T.S.; de Souza, H.E.A.; Ardisson, J.D.; Oliveira, H.S.; Lorencon, E.; Silva, A.C.; Nascimento, L.L.; Patrocínio, A.O.T.; et al. High Water Oxidation Performance of W-Doped BiVO $_{4}$ Photoanodes Coupled to $\mathrm{V}_{2} \mathrm{O}_{5}$ Rods as a Photoabsorber and Hole Carrier. RRL Sol. 2018, 2, 1800089. [CrossRef] 
11. Liu, X.; Liu, Y.; Su, J.; Li, M.; Guo, L. Facile preparation of $\mathrm{BiVO}_{4}$ nanoparticle film by electrostatic spray pyrolysis for photoelectrochemical water splitting. Int. J. Hydrogen Energy. 2015, 40, 12964-12972. [CrossRef]

12. Zhou, M.; Bao, J.; Xu, Y.; Zhang, J.; Xie, J.; Guan, M.; Wang, C.; Wen, L.; Lei, Y.; Xie, Y. Photoelectrodes Based upon $\mathrm{Mo:BiVO} 4$ Inverse Opals for Photoelectrochemical Water Splitting. ACS Nano 2014, 8, 7088-7098. [CrossRef] [PubMed]

13. Hernández, S.; Thalluri, S.M.; Sacco, A.; Bensaid, S.; Saracco, G.; Russo, N. Photo-catalytic activity of BiVO thin-film electrodes for solar-driven water splitting. Appl. Catal. A Gen. 2015, 504, 266-271. [CrossRef]

14. Luo, H.; Mueller, A.H.; McCleskey, T.M.; Burrell, A.K.; Bauer, E.; Jia, Q.X. Structural and photoelectrochemical properties of $\mathrm{BiVO}_{4}$ thin films. J. Phys. Chem. C 2008, 112, 6099-6102. [CrossRef]

15. Huang, M.; Bian, J.; Xiong, W.; Huang, C.; Zhang, R. Low-dimensional Mo:BiVO 4 photoanodes for enhanced photoelectrochemical activity. J. Mater. Chem. A 2018, 6, 3602-3609. [CrossRef]

16. Trzciński, K.; Szkoda, M.; Sawczak, M.; Karczewski, J.; Lisowska-Oleksiak, A. Visible light activity of pulsed layer deposited $\mathrm{BiVO}_{4} / \mathrm{MnO}_{2}$ films decorated with gold nanoparticles: The evidence for hydroxyl radicals formation. Appl. Surf. Sci. 2016, 385, 199-208. [CrossRef]

17. Nishikawa, H.; Hasegawa, T.; Miyake, A.; Tashiro, Y.; Komasa, S.; Hashimoto, Y. Effect of laser fluence and ambient gas pressure on surface morphology and chemical composition of hydroxyapatite thin films deposited using pulsed laser deposition. Appl. Surf. Sci. 2018, 427, 458-463. [CrossRef]

18. Jeong, S.Y.; Choi, K.S.; Shin, H.; Kim, T.L.; Song, J.; Yoon, S.; Jang, H.W.; Yoon, M.; Jeon, C.; Lee, J.; et al. Enhanced Photocatalytic Performance Depending on Morphology of Bismuth Vanadate Thin Film Synthesized by Pulsed Laser Deposition. ACS Appl. Mater. Interfaces 2017, 9, 505-512. [CrossRef]

19. Han, H.S.; Shin, S.; Kim, D.H.; Park, I.J.; Kim, J.S.; Huang, P.-S.; Lee, J.-K.; Cho, I.S.; Zheng, X. Boosting the solar water oxidation performance of a $\mathrm{BiVO}_{4}$ photoanode by crystallographic orientation control. Energy Environ. Sci. 2018, 11, 1299-1306. [CrossRef]

20. Van, C.N.; Chang, W.S.; Chen, J.; Tsai, K.; Tzeng, W.; Lin, Y.; Kuo, H.; Liu, H.; Chang, K.; Chou, W.; et al. Heteroepitaxial approach to explore charge dynamics across $\mathrm{Au} / \mathrm{BiVO}_{4}$ interface for photoactivity enhancement. Nano Energy 2015, 15, 625-633. [CrossRef]

21. Amoruso, S.; Sambri, A.; Wang, X. Propagation dynamics of a $\mathrm{LaMnO}_{3}$ laser ablation plume in an oxygen atmosphere. J. Appl. Phys. 2006, 100, 013302. [CrossRef]

22. Song, J.; Choi, K.S.; Seo, M.J.; Jo, Y.R.; Lee, J.; Kim, T.L.; Jeong, S.Y.; An, H.; Jang, H.W.; Kim, B.J.; et al. Nonequilibrium Deposition in Epitaxial $\mathrm{BiVO}_{4}$ Thin Film Photoanodes for Improving Solar Water Oxidation Performance. Chem. Mater. 2018, 30, 5673-5681. [CrossRef]

23. Hasabeldaim, E.; Ntwaeaborwa, O.M.; Kroon, R.E.; Motaung, D.E.; Coetsee, E.; Swart, H.C. Effect of PLD growth atmosphere on the physical properties of ZnO:Zn thin films. Opt. Mater. 2017, 74, 76-85. [CrossRef]

24. Jackson, B.D.; Herman, P.R. Vacuum-ultraviolet pulsed-laser deposition of silicon dioxide thin films. Appl. Surf. Sci. 1998, 127-129, 595-600. [CrossRef]

25. Rettie, A.J.E.; Mozaffari, S.; McDaniel, M.D.; Pearson, K.N.; Ekerdt, J.G.; Markert, J.T.; Mullins, C.B. Pulsed Laser Deposition of Epitaxial and Polycrystalline Bismuth Vanadate Thin Films. J. Phys. Chem. C 2014, 118, 26543-26550. [CrossRef]

26. Murcia-López, S.; Fabrega, C.; Monllor-Satoca, D.; Hernández-Alonso, M.D.; Penelas-Pérez, G.; Morata, A.; Morante, J.R.; Andreu, T. Tailoring multilayered $\mathrm{BiVO}_{4}$ photoanodes by pulsed laser deposition for water splitting. ACS Appl. Mater. Interfaces. 2016, 8, 4076-4085. [CrossRef]

27. Prześniak-Welenc, M.; Szreder, N.A.; Winiarski, A.; Łapiński, M.; Kościelska, B.; Barczyński, R.J.; Gazda, M.; Sadowski, W. Electrical conductivity and relaxation processes in $\mathrm{V}_{2} \mathrm{O}_{5}$ nanorods prepared by sol-gel method. Phys. Status Solidi 2015, 252, 2111-2116. [CrossRef]

28. Sambri, A.; Khare, A.; Mirabella, S.; di Gennaro, E.; Safeen, A.; di Capua, F.; Campajola, L.; Scotti, U.; di Uccio Amoruso, S.; Granozio, F.M. Plasma dynamics and cations off-stoichiometry in $\mathrm{LaAlO}_{3}$ films grown in high pressures regimes. J. Appl. Phys. 2016, 120, 225306. [CrossRef]

29. Sambri, A.; Aruta, C.; di Gennaro, E.; Wang, X.; di Uccio, U.S.; Granozio, F.M.; Amoruso, S. Effects of oxygen background pressure on the stoichiometry of a $\mathrm{LaGaO}_{3}$ laser ablation plume investigated by time and spectrally resolved two-dimensional imaging. J. Appl. Phys. 2016, 119, 125301. [CrossRef]

30. Thestrup, B.; Toftmann, B.; Schou, J.; Doggett, B.; Lunney, J.G. A comparison of the laser plume from Cu and YBCO studied with ion probes. Appl. Surf. Sci. 2003, 208-209, 33-38. [CrossRef] 
31. Lv, C.; Chen, G.; Sun, J.; Zhou, Y.; Fan, S.; Zhang, C. Realizing nanosized interfacial contact via constructing $\mathrm{BiVO}_{4} / \mathrm{Bi}_{4} \mathrm{~V}_{2} \mathrm{O}_{11}$ element-copied heterojunction nanofibres for superior photocatalytic properties. Appl. Catal. B Environ. 2015, 179, 54-60. [CrossRef]

32. Bott, A.W. Electrochemistry of Semiconductors. Curr. Sep. 1998, 3, 87-91.

33. Yaw, C.S.; Ruan, Q.; Tang, J.; Soh, A.K.; Chong, M.N. A Type II n-n staggered orthorhombic $\mathrm{V}_{2} \mathrm{O}_{5} / \mathrm{monoclinic}$ clinobisvanite $\mathrm{BiVO}_{4}$ heterojunction photoanode for photoelectrochemical water oxidation: Fabrication, characterisation and experimental validation. Chem. Eng. J. 2019, 364, 177-185. [CrossRef]

34. Su, J.; Zou, X.; Li, G.; Wei, X.; Yan, C.; Wang, Y.; Zhao, J.; Zhou, L.; Chen, J. Macroporous $\mathrm{V}_{2} \mathrm{O}_{5}-\mathrm{BiVO}_{4}$ Composites: Effect of Heterojunction on the Behavior of Photogenerated Charges. J. Phys. Chem. C 2011, 115, 8064-8071. [CrossRef]

35. McDonald, K.J.; Choi, K. A new electrochemical synthesis route for a BiOI electrode and its conversion to a highly efficient porous $\mathrm{BiVO}_{4}$ photoanode for solar water oxidation. Energy Environ. Sci. 2012, 5, 8553-8557. [CrossRef]

36. Santos, W.S.d.; Rodriguez, M.; Afonso, A.S.; Mesquita, J.P.; Nascimento, L.L.; Patrocínio, A.O.T.; Silva, A.C.; Oliveira, L.C.A.; Fabris, J.D.; Pereira, M.C. A hole inversion layer at the $\mathrm{BiVO}_{4} / \mathrm{Bi}_{4} \mathrm{~V}_{2} \mathrm{O}_{11}$ interface produces a high tunable photovoltage for water splitting. Sci. Rep. 2016, 6, 31406. [CrossRef]

37. Trzciński, K.; Gąsiorowski, J.; Borowska-Centkowska, A.; Szkoda, M.; Sawczak, M.; Hingerl, K.; Zahn, D.R.T.; Lisowska-Oleksiak, A. Optical and photoelectrochemical characterization of pulsed laser deposited $\mathrm{Bi}_{4} \mathrm{~V}_{2} \mathrm{O}_{11}$, BICUVOX, and BIZNVOX. Thin Solid Films 2017, 638, 251-257. [CrossRef]

38. Chatchai, P.; Murakami, Y.; Kishioka, S.; Nosaka, A.Y.; Nosaka, Y. Efficient photocatalytic activity of water oxidation over $\mathrm{WO}_{3} / \mathrm{BiVO}_{4}$ composite under visible light irradiation. Electrochim. Acta 2009, 54, 1147-1152. [CrossRef]

39. Santos, W.S.d.; Almeida, L.D.; Afonso, A.S.; Rodriguez, M.; Mesquita, J.P.; Monteiro, D.S.; Oliveira, L.C.A.; Fabris, J.D.; Pereira, M.C. Photoelectrochemical water oxidation over fibrous and sponge-like $\mathrm{BiVO}_{4} / \beta-\mathrm{Bi}_{4} \mathrm{~V}_{2} \mathrm{O}_{11}$ photoanodes fabricated by spray pyrolysis. Appl. Catal. B Environ. 2016, 182, 247-256. [CrossRef]

40. Song, J.; Seo, M.J.; Lee, T.H.; Jo, Y.-R.; Lee, J.; Kim, T.L.; Kim, S.-Y.; Kim, S.-M.; Jeong, S.Y.; An, H.; et al. Tailoring Crystallographic Orientations to Substantially Enhance Charge Separation Efficiency in Anisotropic $\mathrm{BiVO}_{4}$ Photoanodes. ACS Catal. 2018, 8, 5952-5962. [CrossRef]

41. Zhang, W.; Wu, F.; Li, J.; Yan, D.; Tao, J.; Ping, Y.; Liu, M. Unconventional Relation between Charge Transport and Photocurrent via Boosting Small Polaron Hopping for Photoelectrochemical Water Splitting. ACS Energy Lett. 2018, 3, 2232-2239. [CrossRef]

42. Zhang, W.; Yan, D.; Tong, X.; Liu, M. Ultrathin Lutetium Oxide Film as an Epitaxial Hole-Blocking Layer for Crystalline Bismuth Vanadate Water Splitting Photoanodes. Adv. Funct. Mater. 2018, 28, 1705512. [CrossRef] 